\title{
Multi-Temporal satellite imagery for infrastructure growth assessment of Dubai City, UAE
}

\author{
Mohamad Ali Khali1 ${ }^{1}$, Rami Al-Ruzouq ${ }^{1}$, Khaled Hamad $^{1}$,and Abdallah Shanableh ${ }^{1}$ \\ ${ }^{1}$ Sustaimanle Civil Infrastructure Systems Research Group, University of Sharjah, Sharjah City, \\ United Arab Emirates
}

\begin{abstract}
Throughout the past few decades, Dubai City has witnessed massive growth in its urban area and infrastructure facilities. The discovery of oil and gas in the Emirate significantly played a role for such a rapid growth. Given this fact and the short time-period for such an expansion, it is crucial to develop an understanding of the patterns of the development in the City, where policy-makers, researchers, and concerned authorities would gain better vision and strategy for the future. Recent advances in satellite imagery in terms of improved spatial and temporal resolutions are allowing for efficient identification of change patterns and the prediction of areas of growth. This study aims to quantify and analyse the spatialtemporal urbanization that took place in Dubai City throughout the past decades (specifically from early 1970's until 2015). Multi temporal satellite images with various geometric and radiometric resolutions will be utilized for this purpose. The suggested methodology consists of a sequence of image processing techniques that include supervised and unsupervised classification.Subsequently, the classified images were utilized to quantify the urbanization of the City. The results show that since 1970 , the urbanization and population have been dramatically increasedby 5 and 12 times respectively. The resulting trend can be potentially usedto evaluate the consequences of massive urban development, such as City infrastructure, water, environmental and the social impact.
\end{abstract}

\section{Introduction}

Over the last few decades, the United Arab Emirates and Dubai City in particular have witnessed significant economic growth since the oil discovery in the region. Such a growth resulted in a rapid urbanization across the City, where a lot of residential, commercial, sports and tourism projects have been implemented. This expansion can be observed by many of the mega scale world's landmarks such as Burj Khalifa (the tallest building in the world), Dubai Mall (the largest mall in the world by the total area), and Miracle Garden (the world's largest natural flower garden). The previous fact can be clearly noticed by observing that approximately $25 \%$ of the world's construction cranes are located in Dubai

*Correspondingauthor:mkhalil@sharjah.ac.ae 
[1]. The development extends to include land and coastal areas where artificial islands were constructedsuch as the Palm Jumeirah, the Palm Deira and the World's Islands.

The present research aims to quantify and analyze the spatial-temporal aspects of the infrastructure growth in Dubai City. In this study, Geographic Information System (GIS) and Remote Sensing (RS) techniqueswill be utilized to detect and quantify urban development in Dubai City. The last 40 years has witnessed remarkable advances in remote sensing and GIS technologies (i.e., satellites' sensor, and image processing and analysis algorithm) [2]-[5]. Refereeing to literature and previous studies, ithas been found that GIS and RS techniques were not used efficiently for change detection and urban development for the study area under consideration. Therefore, the main objectives of this research are as follow:

- Inspect, quantify, and assess the spatial-temporal urbanization growth in Dubai City, and

- Correlate the results from the previous step to the population growth of the City.

This paper is formatted in six sections, as follows:It starts with an introduction (which is this section) to explain the motivation and objectivesof this research. Section two (i.e., theatrical background and literature review) describe few basic concepts of change detection, and illustrate few techniques that can be used to perform it. Further, it reviews list of previous research that were conducted on change detection. Section three discuss the selected study area and few attributes about it. Section four (i.e., data collection and preprocessing) presents the data collection effort in details and the pre-processing steps need to be done before applying change detection to the study area (i.e., Dubai City). Section five (i.e., change detection) is split into twofold. First fold would explain the algorithm of the utilized image classification technique. On the other hand, the second fold will present and discuss the results of the quantification of urban development.Finally, section six will be concluding the research by illustrating on the learned lessons, and state few limitation of the presented research.

\section{Theoretical background and literature review}

Change detection can be defined as the process of identifying differences in a state of an object (or phenomenon) by observing it at different times [6]. It involves the ability to quantify changes using multi-spectral, multi-temporal and/or multi-source imagery having different resolution captured at different times. Traditional change detection techniques are based on visual (in other words manual) comparison of temporal imagery. However, the increasing availability and complexity of accessible imagery makes the visual change detection very hard, and even impractical. Thus, the need of the adoption (and sometimes even development) of an automotive, reliable, and fast change detection techniques is obvious. Several advanced change detection techniques have been developed previously by other scholars [6]-[9]. Example of those techniques are Canny, Prewitt, Roberts, and fuzzy logic [10].

Many research utilized satellite images to detect spatial and temporal changes of objects (e.g. cites or lands) - keeping in mind the different criteria and tools that were used. For instance, Nassar et el. quantified the land cover change in neighboring Dubai City using time series of remotely sensed data for the period between 1972 to 2011 [1]. Their results indicated that a dramatic increase in the expansion of Dubai's urban area has occurred. Specifically, the compound annual growth rate over the whole study period was $10.03 \%$, where the peak growth happened at a rate of $13.03 \%$ during 2003-2005. Furthermore, they noted that the urban growth of Dubai included substantial increase in the vegetation and water bodies, as well as extraordinary rate of construction of offshore islands. Their study 
provided novel insights into the pace and process of urban growth in Dubai. Just as important, the authors noted the importance to evaluate the environmental consequences of this form of rapid urban development in future research. Nasser et al. research and our present study are considered one of the few studies in this topic for Dubai.

$\mathrm{Ji}$ et al. studied the spatial and temporal distribution of expressway and its relations to land cover and population in Beijing City, China [3]. They collected data of roads, land cover and population from 2000 to 2010 . Their results showed that the transfer of land cover near expressways was more intensive and frequent compared to parts of the City. Further, these regions also witnessed the most rapid rates of population growth. An interesting point the authors highlighted is the environmental pollution and health risks to residents living nearby expressways.

In a similar context, Al-sharif et al. studied the spatio-temporal urban and population growth in Tripoli City, Libya using remotely sensed data and Geographic Information System (GIS) [5]. They collected four-satellite-imageries and population censuses of Tripoli for the period from 1987 to 2010 . The study findings demonstrated that there is an obvious indication of urban dispersion and urban sprawl for the metropolitan of Tripoli City, i.e. sprawl is increasing with time. The authors came up with models to give guidance to identify and quantify the change likely to happen if the historical trends of urban growth continue.

$\mathrm{Yu}$ and $\mathrm{Ng}$ combined remote sensing images, landscape metrics and gradient analysis to analyze and compare spatial and temporal dynamics of the urban sprawl in Guangzhou, China [11]. Their results showed that the spatial landscape change in Guangzhou exhibits different patterns at its urban center compared to its rural areas. Moreover, the recent urbanized areas showed a higher fragmentation than the other areas. In terms of characterizing the urban expansion, they found that the urban area expanded towards the north and south of their city. They attributed this to the population increase and rapid economic development. Moreover, they emphasized in their study that in order to reveal the complexity of landscape pattern, temporal data and spatio-temporal dynamics of landscape changes are needed along with the gradient analysis to capture the baseline.

Aljoufie et al. analyzed the spatial urban growth and transportation in Jeddah City, Saudi Arabia using temporal satellite images ranging from 1964 to 2007 [12]. To do so, they developed eight urban growth and transportation indices to quantify the urban growth and transportation patterns in the City of Jeddah. Based on their results, they concluded that urban growth in Jeddah is having an outward expansion and sprawl development. Moreover, they claim that their results improve the understanding of the relationship between the urban growth and transportation infrastructure in the City.

In general, and as illustrated from the previously reviewed paper; the use of GIS and RS is well practiced to detect changes for large-scale study areas. In fact, different techniques, approaches, and indiceswere developed and utilized by others to accomplish the task in the best manner. However, our local area still lacks the saturation of such kind of studies, especially Dubai City - which is as indicated earlier, one of the fastest growing cities around the world.

In this study, we utilized a supervised and unsupervised classification image technique to detect temporal changes for Dubai City over the last 40 years.Given the characteristics and nature of the urbanization that took place in Dubai, it is understandably that the outcome of this research shall presents valuable information not only for Dubai's officials (policy makers, researchers, and concerned authorities), but extend to benefit other governmental and research authorities around the world. 


\section{Study area}

The proposed algorithm in this paper will be applied to the entire City of Dubai (including the offshore development). Figure 1presents the selected study area at the year of 2015 (the image was retrieved from Landsat Satellite, and then clipped to highlight the boundary of the City only).

Dubai is one of the seven emirates that forms the United Arab Emirates, UAE. Dubai (as an Emirate) is considered the second largest Emirate after the Emirate of Abu Dhabi (in terms of both population and area). However, Dubai as a City is actually the largest City in the UAE with the highest population. Dubai has a creek that runs south from the Arabian Gulf for $13 \mathrm{~km}$, dividing the city into Deira to the east and Bur Dubai to the west. As for the climatological aspect, Dubai, and UAE in general, are characterized as a hyperarid climate. In other words, its land is characterized as dry with low rainfall rates (average yearly rainfall is $120 \mathrm{~mm}$ ). Above that, evaporation rates are high (the weather temperature in the City can rise more than $45^{\circ} \mathrm{C}$ in summer the period). The humidity is also high; the average humidity throughout the year is around $60 \%$.

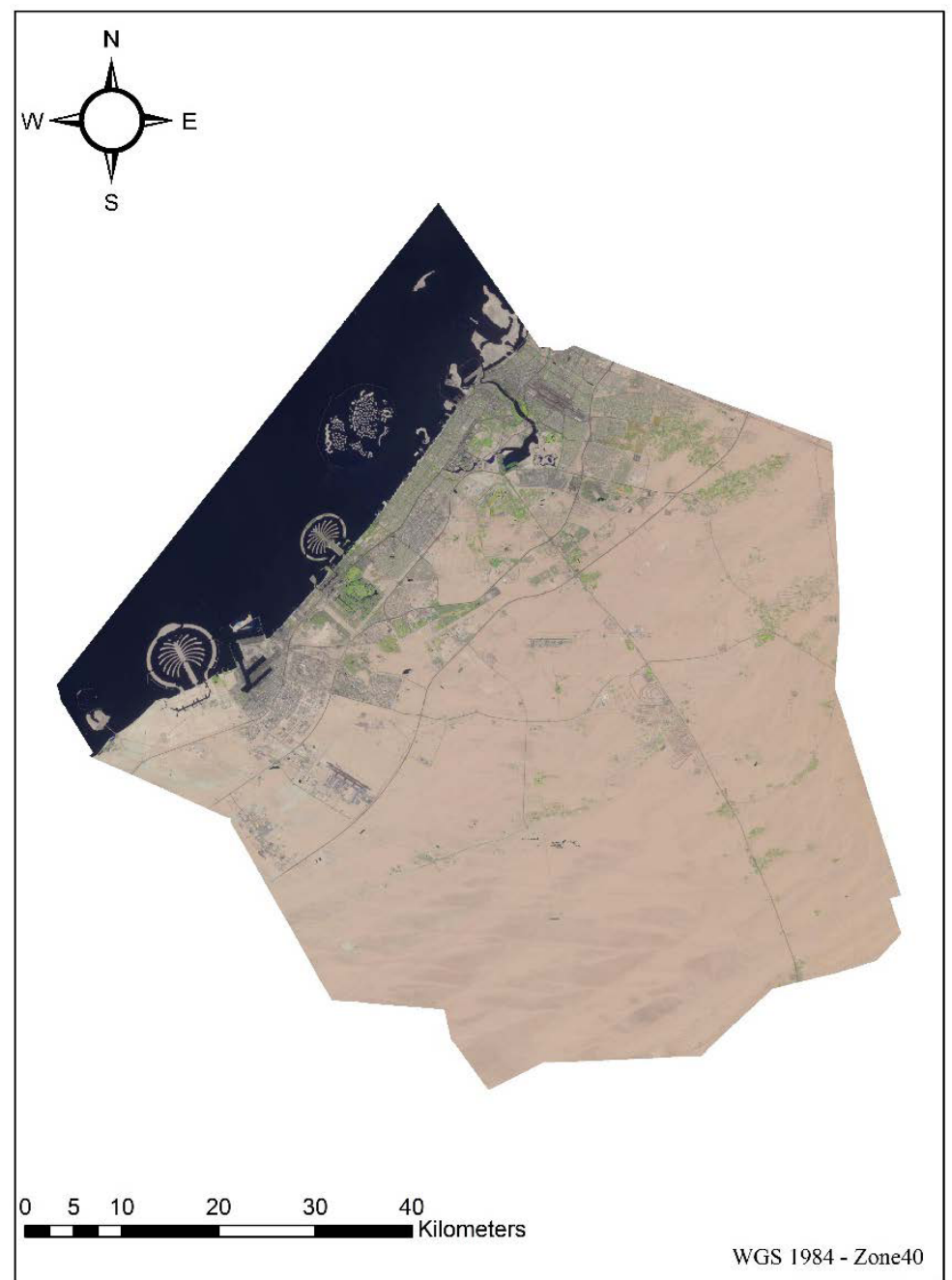

Fig. 1. Dubai City at the year of 2015 - acquired from Landsat8. 


\section{Data collection and processing}

Figure 2 shows the designed flow chart for this research. At a glance, the very first step we needed to do is to collect multi-temporal images for Dubai City form RS satellite. The main source for the images for this research was Landsat from the United States Geological Survey (USGS). After acquiring the images, a pre-prepared mask was used to crop the images for the selected study areas (i.e., Dubai City). It is worth mentioning here that all the processing was done using ArcMap $\subset$. Subsequently, the prepared images were usedto quantify the changes that occurred throughout the years. Moreover, and whenever possible, the population growth will be presented in conjunction to the urbanization growth. Finally, and at the conclusion section, the results will be summarized.

Table 1 shows a summary of the collected satellite images that have different radiometric and geometric resolutions. The table shows the satellite name and the images' acquired years. Figure 3 presents the prepared images that were used later for the supervised and unsupervised classification.

Table 1 : Collected images.

\begin{tabular}{|c|c|c|}
\hline Satellite Name & Year Acquired & Source \\
\hline L1-5 MSS & 1973 & USGS \\
\hline LT 4-5 TM & 1985 & USGS \\
\hline LT 4-5 TM & 1995 & USGS \\
\hline Landsat 7 & 2003 & USGS \\
\hline Landsat 8 & 2015 & USGS \\
\hline
\end{tabular}

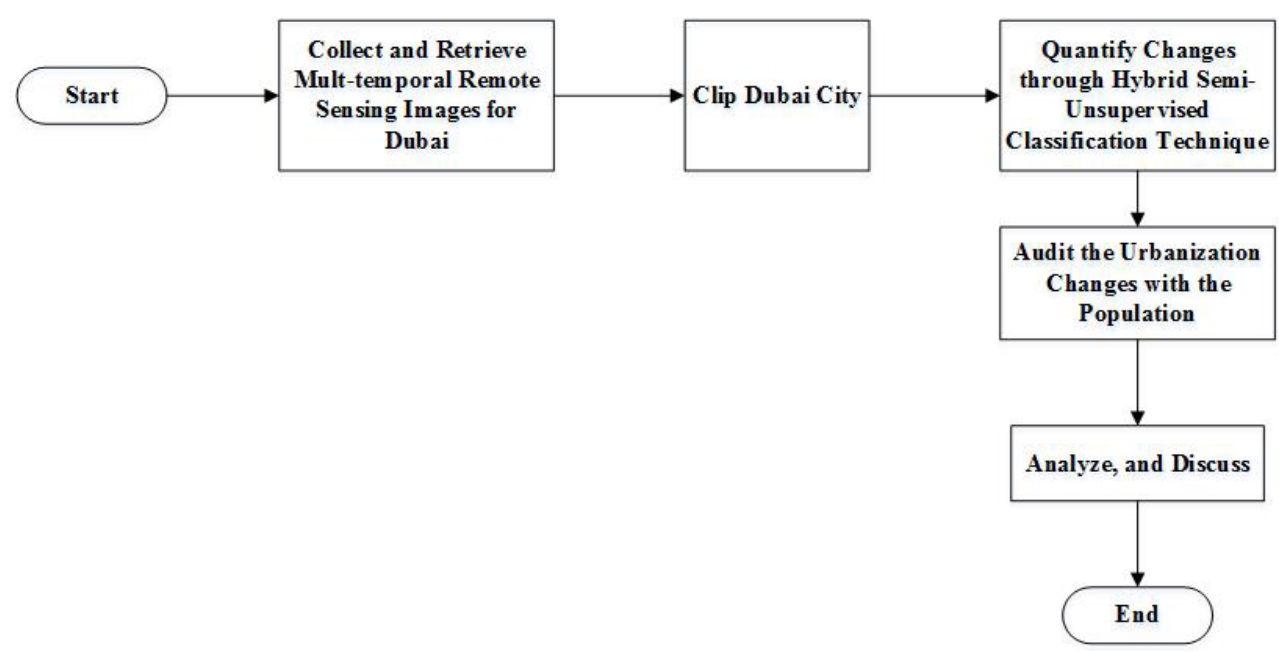

Fig. 2. Research flow chart. 


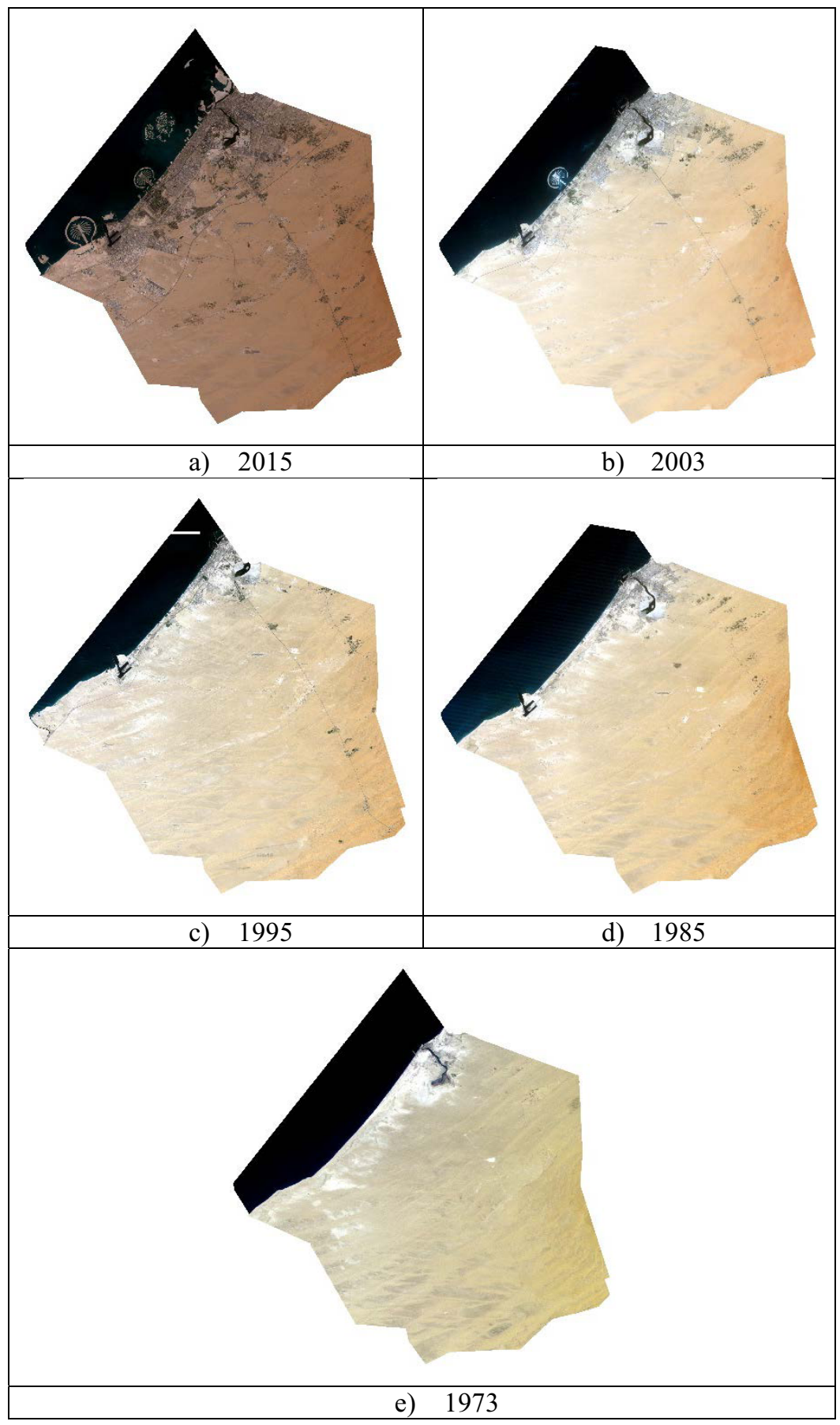

Fig. 3. Multi-temporal images for the study area. 


\section{Change detection}

This section is divided into two parts, first explains the adopted algorithm to classify the image (i.e., the semi-supervised classification-SUC) and second, presents and discuss the outcome results of the urbanization quantification of the classified images.

\subsection{Semi-Unsupervised Classification, SUC}

Digital image classification simply means grouping pixels to represent land cover features. Land cover could be sand, vegetation, urban, or any other types of features. Mainly, there are three techniques to do that, which are: 1) supervised image classification, 2) unsupervised image classification and finally 3) object-based image analysis.

In a nutshell, the difference between the supervised and unsupervised image classification is that the supervised classification asks the user (which is you) to build a training database that contains representative sample of all features the user wants to classify. On the other hand, the unsupervised classification groups features based on the reference properties of pixels. In other words, the user is not supposed to interfere at all in the classification - in contrast to the supervised classification. Finally, the object-based image analysis is an advanced method used to segment a pixel-based image into map objects that can then be classified as a whole. This type of analysis is ideal for mapping with high-resolution imagery. For more information about the three types of classifications, readers are encouraged to explore the following references: [13]-[18]

In this research, we applied a hybrid classification technique that fuses supervised and unsupervised classification. We called that method as "Semi-Unsupervised Classification, $S U C$ ". In specific, the natural breaks (Jenks) method was utilized to classify the images (unsupervised) into three classes, which are, sand and vegetation, water, and urbanization. The images were audited later visually by the user to correct any pitfall that might occur because of radiometric error and/or distortion (supervised interference).Other than this, the remaining steps were done without the intervention of the user. It worth noting here that the result of the unsupervised classification images were converted to vector shapefile to make them amendable - for the supervised intervention, if needed. For that, the method was called "semi".Figure 4 illustrates the flowchart of the proposed technique.

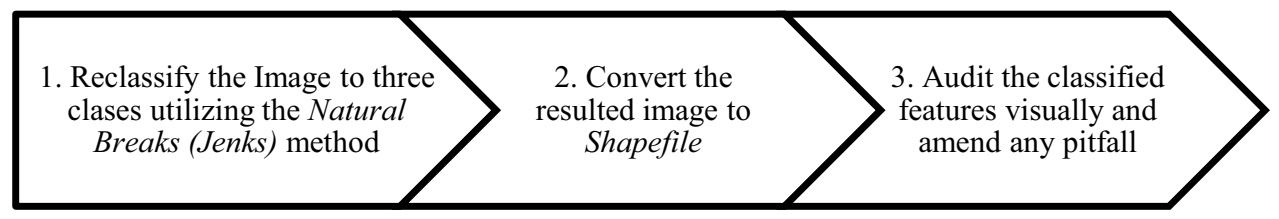

Fig. 4. Semi-Unsupervised Classification flow chart.

\subsection{Results and discussion}

Figure 5 shows a sample result of the 2015 and 1995 semi-unsupervised classified images. As can be seen in the Figure, the SUC was more or less capable of capturing the urbanization trend of the City. Moreover, it is worth emphasizing here that the corrected features by the user were minimal. 


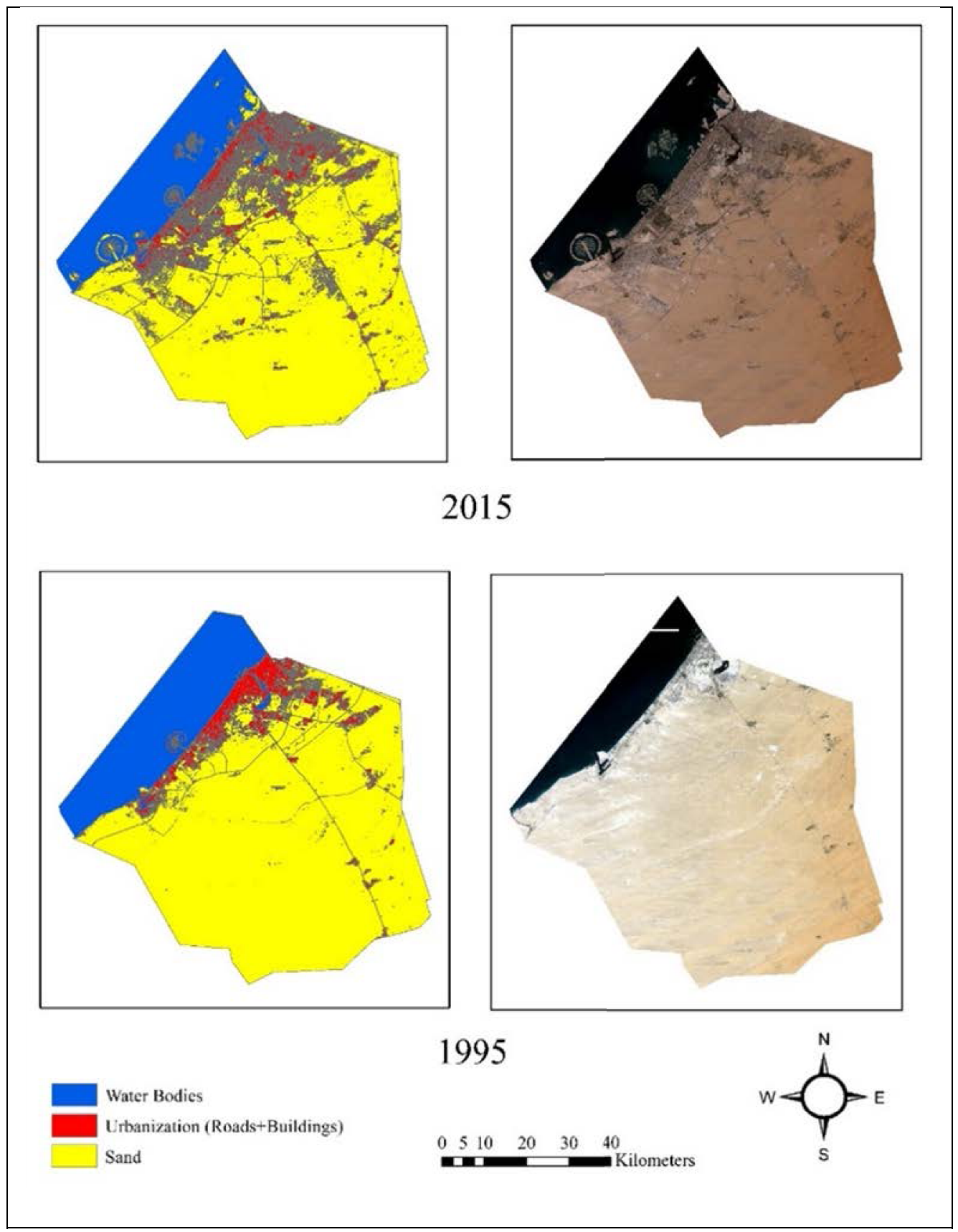

Fig. 5. 2015 and 1995 SUC for Dubai City 
To further our analysis quantitatively; we obtained the urbanization area from the classified images and present it in conjunction to the population growth of the City. Moreover, we opted the Annual Urban Spatial Expansion Index (AUSEI) that was proposed by [12]. The AUSEI would describe the temporal changes of Dubai in terms of its annual urbanization growth rate. The AUSEI is computed as follows:

$$
A U S E I_{t}=\frac{\left(U_{t}-U_{t-1}\right) / U_{t}}{\left(N_{t}-N_{t-1}\right)} \times 100
$$

Where, $U_{t}$ and $U_{t-1}$ are the total urbanized areas at the current year under study and former year; and $\mathrm{N}$ is the total number of years from time $\mathrm{t}$ (current year) to time $\mathrm{t}-1$ (former year).

Table 2 and Figure 6 show how the City evolved in terms of area, AUSE, and population.

Table 2 : Dubai city development during the last 45 years-SUC.

\begin{tabular}{|c|c|c|c|c|}
\hline \multirow{2}{*}{ Year } & \multirow{2}{*}{ Urbanization $\mathbf{( K m}^{\mathbf{2}}$ ) } & \multicolumn{2}{|c|}{ AUSEI } & \multirow{2}{*}{ Population [19] and [20] } \\
\cline { 3 - 5 } & & Period & Index Value (\%) & $2,446,675$ \\
\hline 2015 & 661.0932 & $2003-2015$ & 4.0 & $1,141,610$ \\
\hline 2003 & 343.4742 & $1995-2003$ & 6.0 & 689,424 \\
\hline 1995 & 177.9633 & $1985-1995$ & 1.4 & 370,791 \\
\hline 1985 & 153.5247 & $1973-1985$ & 2.9 & $183,000 @ 1975$ \\
\hline 1973 & Less than 100 & - & - & \\
\hline
\end{tabular}

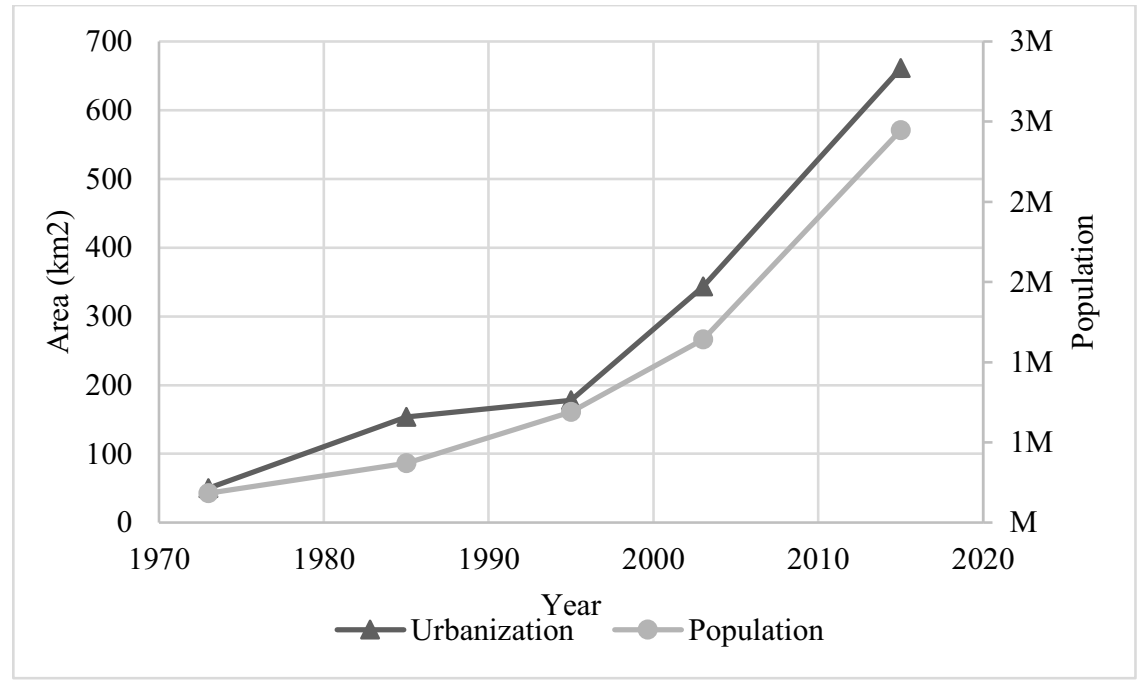

Fig. 6. Urbanization and population growth of dubai city

Out of those results, the following points were drawn out:

- Overall, Dubai City has witnessed a substantial expansion of its urban area during the period 1973 to 2015 . The urban area increased from less than $100 \mathrm{Km}^{2}$ in 1973 to more than $600 \mathrm{Km}^{2}$ in 2015, which is an increase of 500\% in less than 50 years.

- In terms of the AUSEI, the index shows that the highest urbanization pace occurred at the period between 1995 and 2003 (i.e., AUSEI equals to 6\%). In 
contrast, the lowest pace happened during the period of 1985 and 1995, AUSEI of $1.4 \%$. As for the period of 2003 to 2015 , though the world witnessed a harsh economic crisis, the City maintained a moderate urbanization rate, i.e., AUSEI of $4 \%$.

- $\quad$ The population of the City jumped from 183,000 at 1975 to more than $2 \mathrm{M}$ in 2015, which is an increase of more than $1,200 \%$ in less than 50 years.

- As can be seen in Figure 6, the population and urbanization trends almost coincide perfectly to each other. In some other Cities, the trend between both might differ at a certain point of time because of horizontal expansion of those cities - that results from high-rises buildings. Indeed, Dubai City has many high-rise buildings; yet, such a differentiation does not appear to occur in our results. This actually entice us to further investigate this matter in future studies.

- In summary, the results of this analysis highlight the massive development of Dubai City in both urbanization and population aspects during the past four decades. It is worth noting here that one of the major drawback of this approach is that it only demonstrated the overall urbanization of the City. In other others, it could not differentiate between growth in buildings and roads. It might be important to have this detailed differentiation in more advanced studies.

\section{Conclusions}

This study is one of the rare studies that attempted to quantify the rapid urbanization of the City for the last four decades. This was achieved by using different types of Landsat satellite images, such as Landsat 8, and Landsat 7. This effort would be much difficult without the utilization of such remote sensing images. In this study, one approaches was attempted to quantify such changes (though there are multiple in the literature). The method basically is a hybrid unsupervised classification approach (we called it semi-unsupervised classification), where the user has the flexibility to audit the resulted classification, and amend any pitfalls that could occur regarding the any color distortion of the image.

Generally, there was a rapid pace of urbanization development over the entire study period, especially in the late 90's and beginning of the 2000's - regarding Dubai City. And despite the economic crises that occurred in 2007/8, Dubai City continued to evolve but in a slower rate. By comparing the urbanization quantitative analysis with the population growth of the City, we found there was a high correlation trend between both (see Figure 6 ). This is a little bit strange results to us, since we were expecting a small differentiation at a certain point time because of the horizontal expansion of the City (e.g. high-rise buildings). Indeed, this outcome shall open the door for further research to study this matter.

Despite the appealing results presented in this study, there are certain limitations the authors did not consider. The following points illustrate the main limitation in this study:

- There was no ground truth the researchers relied on to validate the attempted approaches,

- The utilized approach (the semi-unsupervised classification) did not separate between roads and buildings development. Such a differentiation might be needed to special micro-case-studies, and

- The study lacked to correlate between the urbanization rate and the environmental status and dashboards of the City. 


\section{References}

1. A. K. Nassar, G. Alan Blackburn, and J. Duncan Whyatt, "Developing the desert: The pace and process of urban growth in Dubai," Computers, Environment and Urban Systems, vol. 45, pp. 50-62, (2014).

2. H. S. Sudhira, T. V. Ramachandra, and K. S. Jagadish, "Urban sprawl: Metrics, dynamics and modelling using GIS," International Journal of Applied Earth Observation and Geoinformation, vol. 5, no. 1, pp. 29-39, (2004).

3. W. Ji, Y. Wang, D. Zhuang, D. Song, X. Shen, W. Wang, and G. Li, "Spatial and temporal distribution of expressway and its relationships to land cover and population: A case study of Beijing, China," Transportation Research Part D: Transport and Environment, vol. 32, pp. 86-96, (2014).

4. [M. K. Jat, P. K. Garg, and D. Khare, "Monitoring and modelling of urban sprawl using remote sensing and GIS techniques," International Journal of Applied Earth Observation and Geoinformation, vol. 10, no. 1, pp. 26-43, (2008).

5. A. a a Al-sharif, B. Pradhan, H. Zulhaidi, M. Shafri, and S. Mansor, "Spatio-temporal Analysis of Urban and Population Growths in Tripoli using Remotely Sensed Data and GIS," Indian Journal of Science and technology, vol. 6, no. 8, pp. 5134-5142, (2013).

6. A. Singh, "Review article digital change detection techniques using remotely-sensed data," International journal of remote sensing, vol. 10, no. 6, pp. 989-1003,( 1989).

7. I. Dowman, "Automated procedures for integration of satellite images and map data for change detection: the archangel project," International Archives of Photogrammetry and Remote Sensing, vol. 32, pp. 162-169, (1998).

8. R. I. Al-Ruzouq and A. F. Habib, "Linear features for automatic registration and reliable change detection of multi-source imagery," Journal of Spatial Science, vol. 57, no. 1, pp. 51-64,( 2012).

9. L. Bruzzone and D. F. Prieto, "Automatic analysis of the difference image for unsupervised change detection," IEEE Transactions on Geoscience and Remote Sensing, vol. 38, no. 3, pp. 1171-1182, (2000).

10. MathWorks, "Edge Detection." [Online]. Available: http://www.mathworks.com/discovery/edge-detection.html. [Accessed: 03-Aug-2016].

11. X. J. Yu and C. N. Ng, "Spatial and temporal dynamics of urban sprawl along two urban-rural transects: A case study of Guangzhou, China," Landscape and Urban Planning, vol. 79, no. 1, pp. 96-109, (2007).

12. M. Aljoufie, M. Zuidgeest, M. Brussel, and M. van Maarseveen, "Spatial-temporal analysis of urban growth and transportation in Jeddah City, Saudi Arabia," Cities, vol. 31, pp. 57-68, (2013).

13. T. Blaschke, "Object based image analysis for remote sensing," ISPRS Journal of Photogrammetry and Remote Sensing, vol. 65, no. 1, pp. 2-16, (2010).

14. R. C. Weih and N. D. Riggan, "Object-based classification vs. pixel-based classification: Comparitive importance of multi-resolution imagery," The International Archives of the Photogrammetry, Remote Sensing and Spatial Information Sciences, vol. XXXVIII, pp. 1-6, (2010).

15. J. Norton, D. Gallant, and N. Scotia, "Supervised Image Classification," no. March, (2014).

16. G. M. Foody and A. Mathur, "Toward intelligent training of supervised image classifications: Directing training data acquisition for SVM classification," Remote Sensing of Environment, vol. 93, no. 1-2, pp. 107-117, (2004).

17. T. W. Lee and M. S. Lewicki, "Unsupervised image classification, segmentation, and enhancement using ICA mixture models," IEEE Transactions on Image Processing, vol. 11, no. 3, pp. 270-279, (2002). 
18. A. E. M. Omran A. Salman, "Differential evolution methods for unsupervised image classification," Proc. of the IEEE Congress on Evolutionary Computation, vol. 2, pp. 966-973, (2005).

19. Dubai Statistical Center, "Population-Clock@www.dsc.gov.ae.”[Online]. Available: https://www.dsc.gov.ae/en-us/EServices/Pages/Population-Clock.aspx.

20. Archived from Tedad.ae, "background @ web.archive.org." [Online]. Available: https://web.archive.org/web/20100516221856/http://www.tedad.ae/english/about_cens us/background.html. 